\title{
Fundamental Way of Charge Formation and Relation between Gravitational Field and Electromagnetic Field
}

\author{
Mukul Chandra Das ${ }^{1 *}$, Rampada Misra ${ }^{2}$ \\ ${ }^{1}$ Singhania University, Rajasthan, India \\ ${ }^{2}$ Department of Electronics, Vidyasagar University, Paschim Midnapur, India \\ Email: " mukuldas.100@gmail.com
}

Received December 28, 2011; revised February 27, 2012; accepted March 8, 2012

\begin{abstract}
A complex motion and complex momentum due to relativistic phenomenon has been deduced in this paper. This procedure leads to explain the generation of a field which is the result of energy momentum complexity (tensor). In this work, a form of complex momentum of photon has been derived. This momentum reveals the construction of electromagnetic field. These procedures have been applied to explain the electromagnetic field of fundamental charged particle and leads to the assumption of fundamental charge. In this works trial would be made to derive a relation between gravitational field and electromagnetic field.
\end{abstract}

Keywords: Superimposed Spins; Complex Momentum

\section{Introduction}

In the theory of relativity, mass velocity relation gives a large quantity of kinetic energy attributed to the particle of rigid configuration [1]. Again this expression (concept) would be same for a spinning particle of rigid configuration as in [2]. A particle can possess two or more superimposed motions to the view of an observer. In [3] Chandrau Iyer and G. M. Probhu describe a constructive method for the composition of two planar boosts with velocities $u$ and $v$ resulting in a velocity $w$. Composition of three linear velocities $u, v$ and $w$ in different directions resulting in a single velocity has been developed in [4]. This leads to the assumption that a body can possess three simultaneous superimposed rotational or spin motions. In this paper, first, trials would be made to derive the energy momentum characteristic of a particle having two superimposed motions. An electromagnetic mass was possible due to Einstein [5]. Einstein strongly believed that field is the form of energy momentum tensor. A charged particle contains electromagnetic field. Again, photons contain electromagnetic field and also electric charge as in [6,7]. In this paper trial would be made to find out the complex characteristic of photon and following this consideration trial would be made to find out the source of fundamental charge of an elementary particle. Gravity is the four dimensional interaction. It is the space-time curvature, basically stress energy tensor. The principles of General Relativity imply that

\footnotetext{
"Corresponding author.
}

gravity and electrodynamics affect each other. According to Einstein all forces of nature are rooted in gravity. He dreamed of unification in between gravity and electrodynamics in much the same way as electricity and magnetism had been combined by Maxwell and Lorenz. But his unified field theory was not particularly successful. Following the general relativistic approach unified field theory has been developed. In [8] it is said that change of both electric and gravitational field results in the creation of a magnetic field in the region of space time which has a dual electro-gravitational nature. Change in magnetic field results in the creation of both electric and gravitational field. Rotating gravitational masses generate magnetic field $B$ and which generates electric field $E$ and gravitational field $G$. In this work trial would be made to derive the unification of gravity and electrodynamics following the combination of electricity and magnetism.

\section{Complex Momentum of a Particle}

For composition of three velocities $(u, v$ and $w)$ we may visualize six inertial frames as discussed in [4] where, co-ordinate of an event from frame $S$ to frame $S_{5}$ would be

$$
H=N_{i j} K
$$

Inverse of this operation would be

$$
K=M_{i j} H
$$

Considering four inertial frames $S, S_{1}, S_{2}$ and $S_{3}$ where, velocity $w=0$ and angle $\psi=0$ in [4] then 
matrix $M_{i j}$ and $N_{i j}$ respectively turn out as

$$
\begin{aligned}
& \bar{M}_{i j}= \\
& \left(\begin{array}{cccc}
\gamma_{u} \gamma_{v}\left(\frac{u v}{c^{2}}+\cos \theta\right) & \gamma_{v} \sin \theta & 0 & -\gamma_{u} \gamma_{v}(v+u \cos \theta) \\
-\gamma_{u} \sin \theta & \cos \theta & 0 & \gamma_{u} u \sin \theta \\
0 & 0 & 1 & 0 \\
\frac{-\gamma_{u} \gamma_{v}}{c^{2}}(v \cos \theta+u) & -\frac{\gamma_{v} \sin \theta}{c^{2}} & 0 & \gamma_{u} \gamma_{v}\left(\frac{u v \cos \theta}{c^{2}}+1\right)
\end{array}\right)
\end{aligned}
$$

$\bar{N}_{i j}=$

$\left(\begin{array}{cccc}\gamma_{u} \gamma_{v}\left(\frac{u v}{c^{2}}+\cos \theta\right) & -\gamma_{u} \sin \theta & 0 & \gamma_{u} \gamma_{v}(v \cos \theta+u) \\ \gamma_{v} \sin \theta & \cos \theta & 0 & \gamma_{v} v \sin \theta \\ 0 & 0 & 1 & 0 \\ \frac{\gamma_{u} \gamma_{v}}{c^{2}}(v+u \cos \theta) & \frac{-\gamma_{u} u \sin \theta}{c^{2}} & 0 & \gamma_{u} \gamma_{v}\left(\frac{u v \cos \theta}{c^{2}}+1\right)\end{array}\right)$

Above conditions imply that Frames $S$ and $S_{1}$ have both their co-ordinate axes aligned and $S_{1}$ is moving at a velocity $u$ along $X_{1}$ axis as observed by $S$. The inertial frame $S_{1}$ has another co-ordinate reference frame $S_{2}$, where $X_{2}$ axis of $S_{2}$, is rotated by an angle $\theta$ counter clockwise with respect to $S_{1}$ on $X_{1} Y_{1}$ plane. Frames $S_{2}$ and $S_{3}$ have both their co-ordinate axes aligned and $S_{3}$ is moving at a velocity $v$ along $X_{3}$ axis as observed by $S_{2}$ [3]. Then magnitude of resultant velocity of an event of $S_{3}$ as observed by $S$ would be

$$
w=\sqrt{\frac{u^{2}+v^{2}+2 u v \cos \theta-\frac{u^{2} v^{2} \sin ^{2} \theta}{c^{2}}}{\frac{u v \cos \theta}{c^{2}}+1}}
$$

In (5) it is clear that $u$ and $v$ are two linear velocities in different directions and rotational angle between $u$ and $v$ is $\theta$. From these implications we get

Case-1: When $\boldsymbol{u}=\boldsymbol{\omega}_{1} \times \boldsymbol{r}$ and $\boldsymbol{v}=\boldsymbol{\omega}_{2} \times \boldsymbol{r}$ as in [4] then it may be called rotation-rotation interaction (i.e. R-R interaction) where, respectively $\omega_{1}$ and $\omega_{2}$ are angular velocities of $S_{1}$ and $S_{3}$ about $X_{1}$ and $X_{3}$ axes. It is also pointed out that origin of frames are same with respect to $S$. If the particle is imagined at the origin of frame $S_{3}$ then it possesses two superimposed spins i.e. spin-spin interaction (S-S interaction) which is homogeneous with R-R interaction.

Case-2: When $\boldsymbol{u}=\boldsymbol{\omega}_{1} \times \boldsymbol{r}$ but $v$ is linear then it may be called rotation-linear (i.e. R-L) interaction and that will be S-L interaction if the particle is present at the origin of $S_{3}$.
Case-3: When $u$ and $v$ both are linear motions then it is called L-L interaction.

Following [9] this may be written as

$$
w=\frac{\sqrt{\boldsymbol{u} \cdot \boldsymbol{u}+\boldsymbol{v} \cdot \boldsymbol{v}+2 \boldsymbol{u} \cdot \boldsymbol{v}-\left(\frac{\boldsymbol{u} \times \boldsymbol{v}}{c}\right) \cdot\left(\frac{\boldsymbol{u} \times \boldsymbol{v}}{c}\right)}}{\frac{\boldsymbol{u} \cdot \boldsymbol{v}}{c^{2}}+1}
$$

So

$$
\boldsymbol{w}=\boldsymbol{a}+\frac{i}{c} \boldsymbol{b}
$$

where, $\boldsymbol{a}=\frac{\boldsymbol{u}+\boldsymbol{v}}{\frac{\boldsymbol{u} \cdot \boldsymbol{v}}{c^{2}}+1}, \quad \boldsymbol{b}=\frac{\boldsymbol{u} \times \boldsymbol{v}}{\frac{\boldsymbol{u} \cdot \boldsymbol{v}}{c^{2}}+1}$ also, $\boldsymbol{a}$ and $\boldsymbol{b}$ are normal to each other.

a represents velocity along space coordinate and $\frac{i}{c} \boldsymbol{b}$ represents velocity along time coordinate. So, $\boldsymbol{w}$ in (7) is a four velocity and from it we get a four velocity matrix $w_{i j}$ where,

$$
w_{i j}=\left(\begin{array}{llll}
w_{x x} & w_{x y} & w_{x z} & w_{x t} \\
w_{y x} & w_{y y} & w_{y z} & w_{y t} \\
w_{z x} & w_{z y} & w_{z z} & w_{z t} \\
w_{t x} & w_{t y} & w_{t z} & w_{t t}
\end{array}\right)
$$

Hence to an observer in $S$ resultant velocity $w$ in (7) is of complex nature. And the relativistic mass of the particle

$$
m=\frac{m_{0}}{\sqrt{1-\frac{w^{2}}{c^{2}}}}
$$

and relativistic Lagrangian would be

$$
L=-m_{0} c^{2} \sqrt{1-\frac{w^{2}}{c^{2}}}
$$

which leads to the form of relativistic momentum

$$
\boldsymbol{P}=\boldsymbol{P}_{1}+\frac{i}{c} \boldsymbol{P}_{2}
$$

where, $\boldsymbol{P}_{1}=\gamma m_{0} \boldsymbol{a}, \quad \boldsymbol{P}_{2}=\gamma m_{0} \boldsymbol{b}$ and $\gamma=\frac{1}{\sqrt{1-\frac{w^{2}}{c^{2}}}}$

when $\theta=90^{\circ}$, then we get from (7)

$$
\boldsymbol{w}=\boldsymbol{u}+\boldsymbol{v}+\frac{i}{c} u v \hat{s}
$$

where, $\boldsymbol{u}, \boldsymbol{v}$ and $\hat{s}$ are mutually normal to each other which leads to the form of momentum

$$
\boldsymbol{P}=m \boldsymbol{u}+m \boldsymbol{v}+\frac{i}{c} m u v \hat{s}
$$


where, $m=\gamma_{o} m_{0}$ and

$$
\gamma_{o}=\frac{1}{\sqrt{1-\frac{1}{c^{2}}\left(u^{2}+v^{2}-\frac{u^{2} v^{2}}{c^{2}}\right)}}
$$

It is seen that Equations (10) and (12) represents a complex momentum of the particle. For a relativistic particle taking $u=v=c$ we get from (11)

$$
\boldsymbol{w}=c \hat{p}+c \hat{q}+i c \hat{r}
$$

For R-R interaction $\hat{p}=\hat{\omega}_{1} \times \hat{r}, \hat{q}=\hat{\omega}_{2} \times \hat{r}$ and $\left(\hat{\omega}_{1} \times \hat{r}\right) \times\left(\hat{\omega}_{2} \times \hat{r}\right)=\hat{r}$.

For R-L interaction $\hat{p}=\hat{\omega}_{1} \times \hat{r}$ and $\hat{q}=\hat{l}$ (i.e. direction of linear velocity of $S_{3}$ )

$$
\left(\hat{\omega}_{1} \times \hat{r}\right) \times \hat{l}=\hat{r}
$$

So, following (12) momentum would be

$$
\boldsymbol{P}^{a}=\frac{E_{k}^{a}}{c} \hat{p}+\frac{E_{k}^{a}}{c} \hat{q}+i \frac{E_{k}^{a}}{c} \hat{r}
$$

It is understood that due to every relativistic motion kinetic energy respective virtual mass $\left(E_{k} / c^{2}\right)$ is attributed to the particle and due to every relativistic spin it rotates about the axis with relativistic velocity approaching that of light [2]. So S-S interaction or S-L interaction or L-L interaction of the particle implies that $E_{k} / c^{2}$ performing two superimposed motions with velocity as in (13) reveals one kind of stress energy tensor (i.e. one kind of field). Following Equation (14) we get momentum-density

$$
\pi=\pi_{1}+i \pi_{2}
$$

So, we can write a function of field $(\varphi)$ as below

$$
\boldsymbol{\varphi}=\zeta\left(\kappa \boldsymbol{\varphi}_{1}+i \rho \boldsymbol{\varphi}_{2}\right)
$$

where, $\pi$ is canonically conjugate to $\varphi$

here, $\varphi_{1}$ and $\varphi_{2}$ are real fields, $\kappa$ and $\rho$ are constants.

\section{Complex Momentum and Field of Photon}

Light is electromagnetic wave. It carries electric and magnetic fields which is proved by Faraday effect and Kerr effects. But photon is a particle whose kinetic energy is $h v$, having mass $h v / c^{2}$. Photon has spin motion about an axis and it may be considered as a small mass $\left(m=h v / c^{2}\right)$ concentrated in a ring of radius $r$ and rotates at velocity of light $(c)$ and it also has linear motion with velocity $c$ along the axis of rotation $[10$, 11]. It is one kind of S-L interaction which is homogeneous with R-L interaction. So resultant velocity would be as in (13) which gives the complex momentum of photon as shown below

$$
\boldsymbol{P}=\frac{h v}{c} \hat{n}+\frac{h v}{c} \hat{l}+i \frac{h v}{c} \hat{r}
$$

This leads to the electromagnetic wave function as specified in $[12,13]$ respectively

$$
\begin{gathered}
\boldsymbol{\psi}=|C| \frac{1}{\sqrt{8 \pi}}(\sqrt{\varepsilon} \boldsymbol{E}+i \sqrt{\mu} \boldsymbol{H}) \\
\left(\Delta-\frac{1}{c^{2}} \frac{\partial^{2}}{\partial t^{2}}\right) \psi+\frac{1}{c^{2}}\left(1-n^{2}\right) \frac{\partial^{2} \psi}{\partial t^{2}}=0
\end{gathered}
$$

where $n=\sqrt{\varepsilon \mu}>1$, Now we can write $\pi_{i j}=S_{i j} / c$ and $\varepsilon^{t}=\frac{1}{2}\left(\varepsilon E^{2}+\frac{1}{\mu} B^{2}\right)$ where, $\pi_{i j}, \varepsilon^{t}$ and $S_{i j}$ are respectively the momentum density energy density and poynting vector of electromagnetic field of photon, So we can write stress energy tensor of this field as

$$
T^{\alpha \beta}=\left[\begin{array}{cccc}
\frac{1}{2}\left(\varepsilon E^{2}+\frac{1}{\mu} B^{2}\right) & S_{x} / c & S_{y} / c & S_{z} / c \\
S_{x} / c & -\sigma_{x x} & -\sigma_{x y} & -\sigma_{x z} \\
S_{y} / c & -\sigma_{y x} & -\sigma_{y y} & -\sigma_{y z} \\
S_{z} / c & -\sigma_{z x} & -\sigma_{z y} & -\sigma_{z z}
\end{array}\right]
$$

where $\sigma_{i j}$ is the Maxwell stress tensor [14]. Since $T^{\alpha \beta}$ is the basis of electromagnetic field so, $\psi$ in (18) would be four dimensional wave function as

$$
\psi_{\alpha}=\left(\begin{array}{l}
\psi_{x} \\
\psi_{y} \\
\psi_{z} \\
\psi_{t}
\end{array}\right)
$$

Again from $[6,7,15]$ a concept is that photon charge is possible with both types, positive and negative, and also upper limit of photon charge is $e_{r} \lesssim 3 \times 10^{-33}$ of elementary charge. It is also possible that photon has two types of spins (i.e. clockwise or anti-clockwise). Since directions of field depend on the direction of momentum so, nature of charges (i.e. positive or negative) would be determined by the type of spins. It is understood that $T^{\alpha \beta}$ reveals the field. Photon carries electric and magnetic fields which are functions of energy $h v$ (or virtual mass $h v / c^{2}$ ) and momentum as given in Equation (17). From the concept of photon we can write energy-momentum tensor due to S-L interaction of a particle which appears as an electromagnetic field. From this we can assume that tensor due to S-S interaction of the particle generates electromagnetic field and the particle would be a rest charged element to the view of an observer in $S$. So a particle of rigid configuration may be charged having electromagnetic field if it possesses S-S or S-L interaction with corresponding relativistic speed. It is to be pointed out that electron, proton carry electric charge as well as electromagnetic field with its rigid configuration. Hence we can write, $\varphi$ in (16) is an electromagnetic 
field function and to make a rest charged particle S-S interaction of it is required.

\section{Field Transformation}

Let gravitational field function in the frame $S_{3}$ in Section 2 be $G_{a}^{\prime}\left(x^{\prime}, y^{\prime}, z^{\prime}, t^{\prime}\right)$ and which would be $G_{a}(x, y, z, t)$ as observed by $S$

where $G_{\alpha}^{\prime}=\left(\begin{array}{c}G_{x}^{\prime} \\ G_{y}^{\prime} \\ G_{z}^{\prime} \\ G_{t}^{\prime}\end{array}\right)$ and $G_{\alpha}=\left(\begin{array}{c}G_{x} \\ G_{y} \\ G_{z} \\ G_{t}\end{array}\right)$

Then using (3) and (4) we get the relation between $G_{\alpha}^{\prime}$ and $G_{\alpha}$ as

$$
G_{\alpha}=\bar{N}_{i j} G_{\alpha}^{\prime}
$$

Inverse of this operation is given as

$$
G_{\alpha}^{\prime}=\bar{M}_{i j} G_{\alpha}
$$

It is understood that a particle of rigid configuration may be charged having S-S or S-L interaction with relativistic speed as in $[2,10]$. So we can write $G_{\alpha}^{\prime}$ performing two superimposed motions (which is associated with $\bar{N}_{i j}$ ) generates electromagnetic field function $\left(\psi_{\alpha}\right)$ which would be homogeneous with $G_{\alpha}$ in (22). So we can consider a relation

$$
\psi_{\alpha}=\Lambda G_{\alpha}
$$

This leads to the form

$$
\psi_{\alpha}=\Lambda \bar{N}_{i j} G_{\alpha}^{\prime}
$$

Similarly, using four velocity matrix as in (7A) we can consider the above relation as

$$
\psi_{\alpha}=\Upsilon w_{i j} G_{\alpha}^{\prime}
$$

where $\Lambda$ and $\Upsilon$ are two constants which depend on the medium.

\section{Conclusion}

A particle can possess two simultaneous superimposed spins (i.e. S-S interaction). To get an electromagnetic field, energy-momentum tensor due to complex motion as well as complex momentum of a relativistic particle as in (7) and (10) are required. But to make an elementary rest charged particle, to the view of an observer, S-S interaction of it is required. In such manners a particle of rigid configuration having gravitational field generates electromagnetic field. Equation (26) reveals the relations between electromagnetic field and gravitational field.

\section{Acknowledgements}

Author thanks the authorities of Satmile High School, Satmile-721452, W. B., India for their continuous encouragements.

\section{REFERENCES}

[1] R. Resnick, "Introduction to Special Relativity," Wiley India Pvt. Ltd., New Delhi, 2009.

[2] M. C. Das and R. Misra, "Relativistic Spin of an Arbitrary Body," Advance Studies in Theoretical Physics, Vol. 6, No. 3, 2012, pp. 135-138.

[3] C. Iyer and G. M. Prabhu, "Composition of Two Lorenz Boosts through Spatial and Space-Time Rotations," Journal of Physical and Natural Sciences, Vol. 1, No. 2, 2007.

[4] M. C. Das and R. Misra, "Three Lorentz Transformations Considering Two Rotations," Advance Studies in Theoretical Physics, Vol. 6, No. 3, 2012, pp. 139-145.

[5] A. Einstein, S. Preuss and A. Wiss, "The Principal of Relativity,” Dover, 1952, pp. 190-198.

[6] V. V. Kobychev and S. B. Porov, "Constraints on the Photon Charge from Observations of Extragalactic Sources," Astronomy Letters, Vol. 31, No. 3, 2005, pp. 147-151. doi:10.1134/1.1883345

[7] C. Sivaram, "Constraints on the Photon Mass and Charge and Test of Equivalence Principle from GRB 990123," Bulletin of the Astronomical Society of India, Vol. 27, No. 4, 1999, pp. 627-630.

[8] V. Y. Kosyev, "Electromagnetic Gravitational Interaction," Conference "Time Machine", Moscow, 12 April 2003.

[9] C. I. Mocanu, "Is Thomas Rotation a Paradox," Apeiron, Vol. 16, 1993.

[10] Y. Rocard, "Thermodynamique," Masson \& Cie, Paris, 1957, p. 250.

[11] C. V. Raman and S. Bhagavantam, "Experimental Proof of the Spin of the Photon," Indian Journal of Physics, Vol. 6, 1931, pp. 353-366.

[12] I. I. Popescu, P. Sterian and M. Dobre, "The Photon Wave Function and the Fresnel Formulas," Romanian Reports in Physics, Vol. 62, No. 2, 2010, pp. 360-368.

[13] R. E. Nistor, "Quantum Aspects of Photon Propagation in Transparent Infinite Homogeneous Media," Romanian Reports in Physics, Vol. 60, No. 3, 2008, pp. 471-491.

[14] J. B. Hartle, “Gravity: An Introduction to Einstein's General Relativity,"3rd Edition, 2009, p. 512.

[15] L. B. Okun, "On the Charge of the Photon," 2005. http://arXiv:hep-ph/0505250v1 